\section{SAT0207 DEPRESSIVE SYMPTOMS IN PATIENTS WITH RHEUMATOID ARTHRITIS IN SARILUMAB TARGET AND MOBILITY TRIALS AND IMPACT OF TREATMENT}

V. Strand ${ }^{1}$, O. Hagino ${ }^{2}$, S. Guillonneau ${ }^{3}$, S. Boklage ${ }^{4}$, M. Reaney ${ }^{5}$, J. Sadeh ${ }^{2}$, N. Narcisse ${ }^{6}$, E. Mangan ${ }^{4}$, T. Kirmura ${ }^{4} .{ }^{1}$ Stanford University, Palo Alto, ${ }^{2}$ Sanofi, Bridgewater, United States, ${ }^{3}$ Sanofi, Chilly-Mazarin, France, ${ }^{4}$ Regeneron Pharmaceuticals, Inc., Tarrytown, United States, ${ }^{5}$ Sanofi, Guildford, United Kingdom, ${ }^{6}$ AIXAL, Boulogne-Billancourt, France

Background: In patients with clinical depression, elevated interleukin-6 (IL-6) levels have been associated with higher symptom severity and greater resistance to standard antidepressant treatments. Depression and IL-6 elevation are highly prevalent in patients with rheumatoid arthritis (RA), and their co-occurrence may have an impact on health-related quality of life (HRQoL). Sarilumab is a human immunoglobulin G1 anti IL-6 receptor $\alpha$ (anti-IL-6R $\alpha$ ) monoclonal antibody for treatment of moderately-to-severely active RA.

Objectives: To explore the effect of sarilumab on HRQoL in patients with moderate-to-severely active RA with co-existing symptoms of depression.

Methods: Post-hoc statistical analyses were performed on the Medical Outcomes Study Short Form 36 (SF-36) in 2 randomized controlled trials, MOBILITY (NCT01061736) and TARGET (NCT01709578), of sarilumab subcutaneous 150 mg or 200 mg every 2 weeks vs placebo, each combined with conventional synthetic disease modifying anti-rheumatic drugs. Patients were classified at baseline for probable major depressive disorder ${ }^{1}$ (PMDD; SF-36 mental health (MH) domain score $\leq 56$ ) or probable depressed mood and anhedonia ${ }^{2}$ (PDMA; score $\leq 10$ on both items of the MH domain: "Have You Felt Downhearted and Depressed" and "Have You Felt So down in the Dumps that nothing could cheer you up"). Analyses of least squares mean differences in changes from baseline in SF-36 domain scores for sarilumab versus placebo in the PMDD and PDMA subgroups were performed at Weeks 4, 12 and 24 for TARGET and Weeks 24 and 52 for MOBILITY. Sensitivity analysis adjusted for baseline Disease Activity Score 28 C-reactive protein (DAS28-CRP).

Results: Of the 546 patients from TARGET and 1197 from MOBILITY, 59.5\% and $60.2 \%$ were classified as PMDD respectively, and $50.4 \%$ and $51.6 \%$ as PDMA. In both RCTs disease duration and baseline DAS-28 CRP, tender and swollen joint count (table 1) and SF-36 domain scores (figure 1) were similar between sarilumab and placebo within the PMDD and PMDA subpopulations. TARGET: MH scores for PMDD and PDMA subgroups were nominally higher $(p<0.05)$ for sarilumab $200 \mathrm{mg}$ versus placebo at all assessments. Both subgroups also scored nominally higher $(\mathrm{p}<0.05)$ in the domains of physical functioning $(P F)$, role-physical $(R P)$, bodily pain $(B P)$, general health $(G H)$, vitality $(V T)$ and social functioning (SF) but not role-emotional (RE) in the PMDD subgroup at Week 24 (figure 1). MOBILITY: all scores except PF and RE were nominally higher $(p<0.05)$ for sarilumab $200 \mathrm{mg}$ versus placebo for Weeks 24 (figure 1). Sensitivity analysis provided similar results. Exploratory results also suggested reduced prevalence of depressive symptoms over the course of the trial.

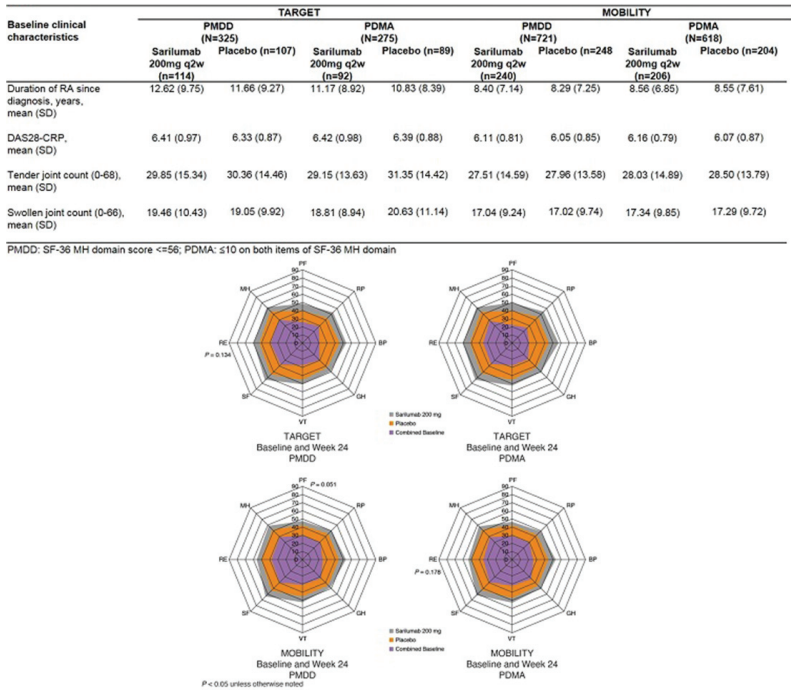

Figure 1. PMDD: SF-36 MH domain score $\leq 56$; PDMA: $\leq 10$ on both items of SF-36 MH domain

Conclusions: In patients with RA and depressive symptoms, sarilumab provided clinically meaningful improvements in most domains of health status/ HRQoL compared with placebo, which may be a function of targeting the IL-6R $\alpha$ and subsequent reduction in disease activity.
REFERENCES:

[1] Matcham, et al. BMC Musculoskeletal Disorders 2016;17:224.

[2] Sun Y, et al. EULAR Congress 2017 June 14-17; Madrid, Spain.

Acknowledgements: Research sponsored by Sanofi and Regeneron Pharmaceuticals, Inc.

Disclosure of Interest: V. Strand Consultant for: AbbVie, Amgen, Biogen, Celltrion, Consortium of Rheumatology Researchers of North America, Crescendo Bioscience, Eli Lilly, Genentech/Roche, GlaxoSmithKline, Hospira, Janssen, Merck, Novartis, Pfizer, Regeneron Pharmaceuticals, Sanofi and UCB, O. Hagino Shareholder of: Sanofi, Employee of: Sanofi, S. Guillonneau Shareholder of Sanofi, Employee of: Sanofi, S. Boklage Shareholder of: Regeneron Pharmaceuticals, Inc., Employee of: Regeneron Pharmaceuticals, Inc., M. Reaney Shareholder of: Sanofi, Employee of: Sanofi, J. Sadeh Shareholder of: Sanofi, Employee of: Sanofi, N. Narcisse Consultant for: Sanofi, E. Mangan Shareholder of: Regeneron Pharmaceuticals, Inc., Employee of: Regeneron Pharmaceuticals, Inc., T. Kirmura Shareholder of: Regeneron Pharmaceuticals, Inc., Employee of: Regeneron Pharmaceuticals, Inc.

DOI: 10.1136/annrheumdis-2018-eular.3723

\section{SAT0208 HOW GOOD ELDERLY RHEUMATOID ARTHRITIS PATIENTS RESPOND AT FIRST YEAR OF TREATMENT WITH CERTOLIZUMAB PEGOL?}

V. Torrente-Segarra ${ }^{1}$, M. Fernandez Prada ${ }^{2}$, R. Expósito ${ }^{3}$, N. Garrido Puñal ${ }^{4}$, A. Sánchez-Andrade ${ }^{5}$, J. R. Lamúa-Riazuelo ${ }^{6}$, A. Olivé ${ }^{7}$, J. V. Tovar ${ }^{8}$ on behalf of RENACER Study Group (REgistro NAcional CERtolizumab). ${ }^{1}$ Rheumatology, Hospital General Hospitalet-Moisès Brogg, Hospitalet Llobregat, ${ }^{2}$ Hospital Universitario de Guadalajara, Guadalajara, ${ }^{3}$ Hospital Comarcal de Laredo, Laredo, ${ }^{4}$ Hospital Universitario Virgen del Rocío, Sevilla, ${ }^{5}$ Complejo Hospital Universitario Lucus Augusti, Alicante, ${ }^{6}$ Hospital Universitario del Henares, Alcalá Henares, ${ }^{7}$ Hospital Germans Tries i Pujol, Badalona, ${ }^{8}$ Hospital General Universitario de Elche, Elche, Spain

Background: In rheumatoid arthritis (RA), the efficacy and safety of Certolizumab pegol (CZP) is well stablished, as reported in randomized clinical trials $(\mathrm{RCT})^{1}$ and some registries ${ }^{2}$. Only the $30 \%$ of RA patients are within the age range of 65 years or older. However, they are usually excluded from the RCT. Aging is associated with declining immune cell function and age-related comorbidities ${ }^{3,4}$.

Objectives: The aim of this study was to determine the effectiveness and safety of CZP in elderly patients in a real world setting at 12 months follow-up.

Methods: Observational longitudinal prospective study of RA patients from 40 sites in Spain. Variables (baseline, 3- and 12-month assessment): socio-demographics, smoking status, previous synthetic DMARD (sDMARD) and biological DMARD (bDMARD) use; TJC, SJC, ESR, CRP, DAS28. Response variables EULAR Moderate/Good Response and DAS28 remission and Safety were assessed. Low Disease Activity as DAS28 remission or mild DAS28. Descriptive, comparative and Logistic regression analyses were performed comparing $<65 \mathrm{vs.}$ $>65$ yr population. Kaplan-Meier survival curve was performed.

Results: A total of 501 RA patients were included, $23 \%$ were aged $>65 \mathrm{yr}$ (mean age $70.8( \pm 4.5 \mathrm{SD}) \mathrm{yr})$. Sociodemographics and baseline features are shown in table 1.

Table 1

\begin{tabular}{llll}
\hline & $<65$ & $>65$ & $\mathbf{P}$ \\
\hline Women & $77.7 \%$ & $80.7 \%$ & NS \\
$\begin{array}{l}\text { Disease evolution } \\
\text { (yr) }\end{array}$ & $6.7( \pm 6.5$ & $10.4( \pm 9.1 \mathrm{SD})$ & $<0.001$ \\
$-\leq \mathbf{2}$ yr & $\mathrm{SD})$ & $-18.3 \%$ & -0.012 \\
Smoking status: & $-30.5 \%$ & & \\
Current & $19.9 \%$ & $9.1 \%$ & 0.024 \\
Exsmoker never & $13.6 \%$ & $11.1 \%$ & \\
Bio-naïve & $66.5 \%$ & $79.8 \%$ & $\mathrm{NS}$ \\
& $56 \%$ & $53.1 \%$ & \\
& & *previous Abatacept use was higher in $>65 \mathrm{yr}$ & \\
& $(\mathrm{p}=0.017)$ & \\
\hline
\end{tabular}

Efficacy variables are shown in table 2.

\begin{tabular}{llll}
\hline Variable & $<65$ years & $\geq 65$ years & $\boldsymbol{p}$ value \\
\hline DAS28 Remission (yes) & $43 \%$ & $31.3 \%$ & 0.026 \\
DAS28 Low Disease Activity & $57.5 \%$ & $47.3 \%$ & $N S$ \\
DAS28 $>$ 1.2 score reduction & $55.4 \%$ & $60.7 \%$ & $N S$ \\
EULAR Response & $67.7 \%$ & $75 \%$ & $N S$ \\
CZP Retention rate & $74.6 \%$ & $67.5 \%$ & $N S$ \\
Adverse Events & $11.3 \%$ & $19.3 \%$ & 0.026
\end{tabular}

